\section{VISUAL ATTENTION}

\section{Splitters and Lumpers}

JANS ET AL. (2010). Visual spatial attention to multiple locations at once: The jury is still out. Psychol Rev, 117, 637.

Cave et AL. (2010). Split attention as part of a flexible attentional system for complex scenes. Psychol Rev, 117, 685.

Can you direct your spatial selective attention to two or more noncontiguous locations at the same time? Much ink has been spilled on this topic over the past few decades, and it seems very timely given our ever-increasing interest in the ability to multitask. The April 2010 issue of Psychological Review contains a massive contribution to this discussion in the form an article by Jans et al. and a response from Cave et al. (and a round of responses to each other). Jans et al. argue that convincing evidence for split attention requires experiments that meet four criteria: (1) The task has to be difficult enough to demand attention, (2) stimuli need to be brief enough to preclude serial shifts of attention, (3) ISIs from cue to target need to be in the right range, and (4) you need to measure the effects of attention in enough locations to assure that no oddly shaped but single-attended region could account for the data. When Jans et al. apply these criteria to a very large set of experiments (their Table 1, listing experiments, runs to $2.5 \mathrm{pp}$.), they find that none satisfy their requirements and conclude that there is no solid evidence for split attention. Moreover, they think that splitting attention, if it can be done, is a bit of a laboratory parlor trick, requiring extensive training, rather than a routine part of attention.

Cave et al. take a different view. They find the four criteria to be somewhat arbitrary and the requirement that experiments meet all four to be overstrict. Moreover, they argue that Jans et al. apply their criteria more strictly to one side of the debate than to the other. Cave et al. see attention as a much more flexible tool that can be used to select one thing, more than one thing, or potentially everything, although they concede that splitting attention will incur costs.

The debate occupies 60 pages, because what seems like a straightforward question about the unity of attention is not straightforward at all. Consider a situation in which you are told to attend to the red letters in a display. Everyone agrees that it is possible to give some sort of boost to each letter in a spatially separated set of red letters, but this is considered "feature," not "spatial," attention in most formulations. Now suppose that, after the color appears, it is taken out of the scene, and attention is probed at formerly red locations. If you find signs of selective attention, is that feature attention, or does it now represent evidence for a splitting of spatial attention? At the risk of muddying the waters still further, perhaps the answer changes as a function of the work that attention is doing. Much of the evidence mustered in this debate comes from simple detection tasks in which the presence of attention is marked by a speeded RT to a simple probe. Very little is said in these studies about the role of attention in object identification. Attention seems to be required when object identification involves the binding of features. That is, if you need to tell the difference between one object with a red vertical part and a blue horizontal part, and another with blue vertical and red horizontal parts, you will need to deploy attention to those objects, and it seems likely that you will need to deploy attention to one object at a time. "But," a splitter might argue, "even if one task requires a single focus of attention, that wouldn't mean that all spatial attention is singular." The debate thus seems likely to continue for some time. However, any future debater will need to attend to the exten- sive scholarship of these two groups of researchers. - J.M.W.

\section{AUDITORY MEMORY}

\section{That Old Familiar Noise}

Agus ET AL. (2010). Rapid formation of robust auditory memories: Insights from noise. Neuron, 66, 610.

To make sense of our auditory world, we must identify sensory patterns that reoccur and retain those sound patterns in memory. From a chickadee's characteristic call to our favorite ring tone, memory for auditory patterns allows us to interact effectively with our environment when encountering familiar sounds and their accompanying sources. Agus et al. investigated memory for complex sounds by presenting listeners with repetitions of random noise, which were necessarily unfamiliar, complex, and meaningless. Agus et al. found that the formation of auditory memories, even for this type of random noise, was exceedingly rapid and robust, with memories for multiple distinct samples of noise retained for several weeks. Listeners engaged in a repetition detection task in which they were presented with trials of continuous random noise containing segments that were or were not repeated. A subset of the repeated segments then occurred again multiple times throughout experimental blocks, giving listeners the opportunity to learn those particular noises. Listeners were extremely sensitive to repeated exposure. As indexed by their increased ability to detect repetitions, auditory memories were formed rapidly with near-ceiling performance within the first several exposures. These memories, in addition to lasting for weeks, were also resilient across certain types of acoustic transformations. These findings suggest that auditory memories are far more robust than has been previously assumed and can be learned rapidly with no supervision 
or feedback. This type of rapid and long-lasting memory for arbitrary auditory patterns may reflect listeners' flexible sensitivity to natural sounds and serve as the basis for sound recognition across a variety of auditory domains. - L.C.N.

\section{SACCADIC ADAPTATION}

\section{Where Things Are Depends on How You Look at Them}

ZimMERMAN \& LAPPE (2010). J Vis, 10(6): Art. 2. doi:10.1167/10.6.2

In a saccadic adaptation procedure, the participant makes repeated saccades to a peripheral target, but during the saccade, the target gets displaced by the same offset on each trial. After adaptation, although the participant's saccades aim at the target's start location, they reach its end location (Noto \& Robinson, 2001, Cogn $\mathrm{Br}$ Res 12:301; Wallman \& Fuchs, 1998, $J$ Neurophys 80:2405). In addition, if the participant is asked to judge the target's start location after making one of these saccades, his or her judgment tends to be shifted toward the end location. It has been proposed that target localization uses an efference copy of the saccade required to fixate the target, and that this efference copy gets interpreted without compensating for the adaptation; as a result, the target's start location gets mapped to the landing location of the saccade (i.e., to the target's end location). But suppose no saccade is actually produced; is the target location then judged veridically? This was the question investigated by Zimmerman and Lappe. After saccadic adaptation, observers were tested in trials in which they maintained continuous central fixation and adjusted probe light locations to match the locations of briefly flashed peripheral targets. The results were striking. If the target end location during adaptation was farther from fixation than the start location, in the test trials, targets were indeed mislocalized, and the localization errors were identical to the changes in saccade length due to adaptation. However, if the target end location was closer to fixation than the start location, although adaptation shortened saccades substantially, the target locations were judged veridically in test trials. Evidently, adaptation that increases the gain of saccades changes the structure of visual space, whereas adaptation that decreases the gain of saccades does not! - C.F.C.

\section{VISUAL-AUDITORY PROCESSING}

\section{The Times That Bind}

FUJISAKI \& NISHIDA (in press). A common perceptual temporal limit of binding synchronous inputs across different sensory attributes and modalities. Proc R Soc B. doi:10.1098/ rspb. 2010.0243

Over the years, vision scientists have assembled a large catalogue of cross-modal phenomena, but in most cases we have not been able to pinpoint the mediating mechanisms. Relatively peripheral channels may integrate auditory and visual stimuli, but we do not need such channels to explain why, for example, a sound on the right induces leftward motion in a horizontal line (Shimojo et al., 1997, Vis Res 37:1575). Fujisaki and Nishida attempted to get less ambiguous evidence for cross-modal channels by asking observers to discriminate between opposite phase relationships in two alternating stimuli. They had to decide, for example, whether a flickering light was red or green when the pitch of a synchronously alternating tone was high rather than low. With each of 13 different stimulus combinations, discrimination was possible at temporal frequencies below $2 \mathrm{~Hz}$, but only the three within-attribute discriminations (e.g., between two flickering lights) were possible at temporal frequencies above $3 \mathrm{~Hz}$. This finding strongly implicates a slow, central binding mechanism that is not selective for modality. Nonetheless, some compelling evidence for cross-modal channels does exist, such as subthreshold summation of luminance and tactile vibration (Arabzadeh et al., 2008, Psychol Sci 19:635). What remains is to reconcile such exceptional findings as this with Fujisaki and Nishida's seemingly robust and intuitively satisfying results. -J.A.S.
VISUAL PERCEPTION

\section{Visual Acuity and Placebo Effects}

LANGER ET AL. (2010). Believing is seeing: Using mindlessness (mindfully) to improve visual acuity. Psychol Sci, 21, 661 .

The well-known placebo effect, thought to arise from the mere expectation that a treatment will have beneficial effects, reveals that the mind can often have a powerful influence on the physical health of the body. Placebo effects have been observed following the ingestion of inert medication for the treatment of a wide range of ailments, including pain, depression, and nausea; following sham surgery for the treatment of arteriosclerosis; and following sham neural stimulation for the treatment of Parkinson's disease. However, despite these examples, I was still surprised to learn that one might see with higher visual acuity simply by embracing a positive outlook on vision.

This form of "placebo vision" is precisely what Langer et al. appeared to produce in a provocative series of experiments. In Experiment 1 , they utilized the expectation that Air Force pilots have excellent vision. Accordingly, participants assigned to the experimental group were asked to embrace the identity of Air Force pilots while flying in a flight simulator, whereas those in the control group were asked to pretend to be Air Force pilots while sitting in a "broken" flight simulator. Although there were no differences in visual acuity before the manipulation (all had $20 / 20$ vision or better), $40 \%$ of the participants in the experimental group showed improved visual acuity following the manipulation, whereas $0 \%$ of the participants in the control group showed improvement. Subsequent experiments controlled for motivation but still showed a similar pattern. In Experiment 2, Langer et al. utilized the expectation that athletes have excellent vision. Accordingly, the experimental group were asked to perform 15 jumping jacks (an activity judged to be more athletic by independent raters), whereas those in 
the control group were asked to skip around the room for $1 \mathrm{~min}$ (an activity judged to be less athletic by independent raters). As in Experiment 1, there were no differences in visual acuity before the experimental manipulation. Although both groups became equally aroused (based on pulse rate) during the experimental manipulation, $37.5 \%$ of the participants in the experimental group showed improved visual acuity following it, whereas only $6.2 \%$ of the participants in the control group showed improvements. Finally, in Experiment 3, Langer et al. utilized the common belief that the letters at the bottom of the Snellen eye chart are harder to read than the letters at the top. If acuity is influenced by this expectation, reversing the order of the lines might result in higher acuity scores. To examine this question, observers read standard eye charts and reversed eye charts from a distance of 10 feet. As expected, the findings showed that observers identified significantly more letters from the smallest and the next-tosmallest lines when the letters were presented in the reverse format than when they were presented in the standard format - a result the authors attributed to expectation.

Before you throw out your eyeglasses, however, note that Langer et al. only reported their results in terms of the number of participants who improved across time or in terms of the number of correct items. Thus, we don't really know whether the increase in accuracy covaried with an increase in response rates, which might simply indicate a more liberal threshold to respond in the experimental group rather than a true improvement in visual sensitivity. - B.S.G.

\section{AUDITION}

\section{Speech Privacy in Meeting Rooms}

Bradley \& Gover (2010). Speech levels in meeting rooms and the probability of speech privacy problems. $J A S A, \mathbf{1 2 7}, 815$.

It goes without saying that in some meetings privacy must be maintained, and the first thing to be sure of is that speech sounds are not audibleor at least not intelligible-outside the room where the meeting is held. Bradley and Gover establish a procedure for estimating the likelihood that speech is intelligible at different points outside of meeting rooms. Their procedure is based on measurements of speech levels for different meetings and meeting rooms. They measured sounds at various moments and at various points near the periphery of the rooms, taking into account room size, whether there was amplification, and the number of occupants. Average speech levels in the periphery were only $2 \mathrm{~dB}(\mathrm{~A})$ higher in rooms with amplification systems than without, and a small effect of room size on average speech levels in meeting rooms was observed only when no amplification system was used. To aid in the use of their findings, the authors provide estimates of speech privacy ratings based on average noise levels outside the room and the transmission characteristics of the room. However, their ratings do not apply in all cases; the investigation did not include, for instance, teleconferences, where participants might speak more loudly than in regular meetings. - S.G.

\section{OBJECT FILES}

\section{Cross-Modal Object Files}

JORDAN ET AL. (2010). See an object, hear an object file: Object correspondence transcends sensory modality. Vis Cogn, 18, 492.

Visual attention does not select raw, unorganized visual information, but instead seems to select objects, which can range from clusters of features that somehow "go together" via grouping principles, to meaningful objects familiar to the perceiver. The object file is a useful concept for understanding how attention tracks and selects objects. An object file is a collection of an object's propertiessuch as its size, color, and locationwhich is updated as an object changes its position. Almost everything known about object files comes from the visual modality. In the widely used object-reviewing task, observers first see a preview of two or more objects (e.g., an "A" to the left of fixation and a " $B$ " to the right) that appear inside placeholder frames. The objects disappear, and the placeholders move to new locations. An object then reappears, and observers recognize the object as quickly as possible. The object can appear inside its original placeholder (a congruent trial) or in the other (an incongruent trial); observers typically are faster to recognize objects on congruent trials than on incongruent trials.

Despite our wealth of knowledge about visual object files, many objects are potentially multimodal, as when a phone rings or a dog barks. In two elegant experiments, Jordan et al. demonstrate that object files store and update abstract, cross-modal information about objects. Using a variant of the object-reviewing task, their observers first saw two visual objects (e.g., a phone to the left of fixation and a dog to the right) that, as is typical for this paradigm, appeared inside placeholders. The objects then disappeared and the placeholders moved. However, a sound was next presented, and observers reported whether the sound corresponded to either of the previous objects (match trials) or to neither (no-match trials). As with the purely visual version of the task, the sounds came from locations either congruent or incongruent with their visual placeholder. Observers were faster to report matching sounds when those sounds appeared in congruent rather than incongruent locations, suggesting that the visual object preview activated auditory information about the visible objects.

Jordan et al.'s results are important in demonstrating that object files can contain information from other modalities. Although much research has demonstrated the cross-modal nature of attention, Jordan et al. show that these cross-modal links need not be indexed by spatial position only. Instead, visual and auditory information might be linked by object familiarity, and information from one modality might activate information in another modality. - S.P.V. 